\title{
ON STARS WITH THE MOST FAVOURABLE BACKGROUND IN OBSERVATIONAL HISTORY
}

\author{
G. VAN HERK \\ Leiden Observatory, The Netherlands
}

The stars in the southern hemisphere with the best observational history in meridian work have been identified and listed at Leiden according to their positions in the sky. Variable and double stars and all stars with $|b| \leqslant 10^{\circ}$ were omitted. The work could be carried out only for the section from $0^{\mathrm{h}}$ to $16^{\mathrm{h}}$ right ascension. The criterion for good observational history was either to have eight or more catalogue entries in the GFH, or to have five or more entries in the Hamburger IdS.

The first group mentioned contains 5109 stars, 4572 of them having some entries in the Hamburger Index as well. In the second group, 698 stars were found, all of them having entries in the GFH. There are 115 stars which meet the criteria for both groups. The numbers of stars occurring in fields 20 minutes wide in $\alpha$ and 0.05 in $\sin \delta$ is shown

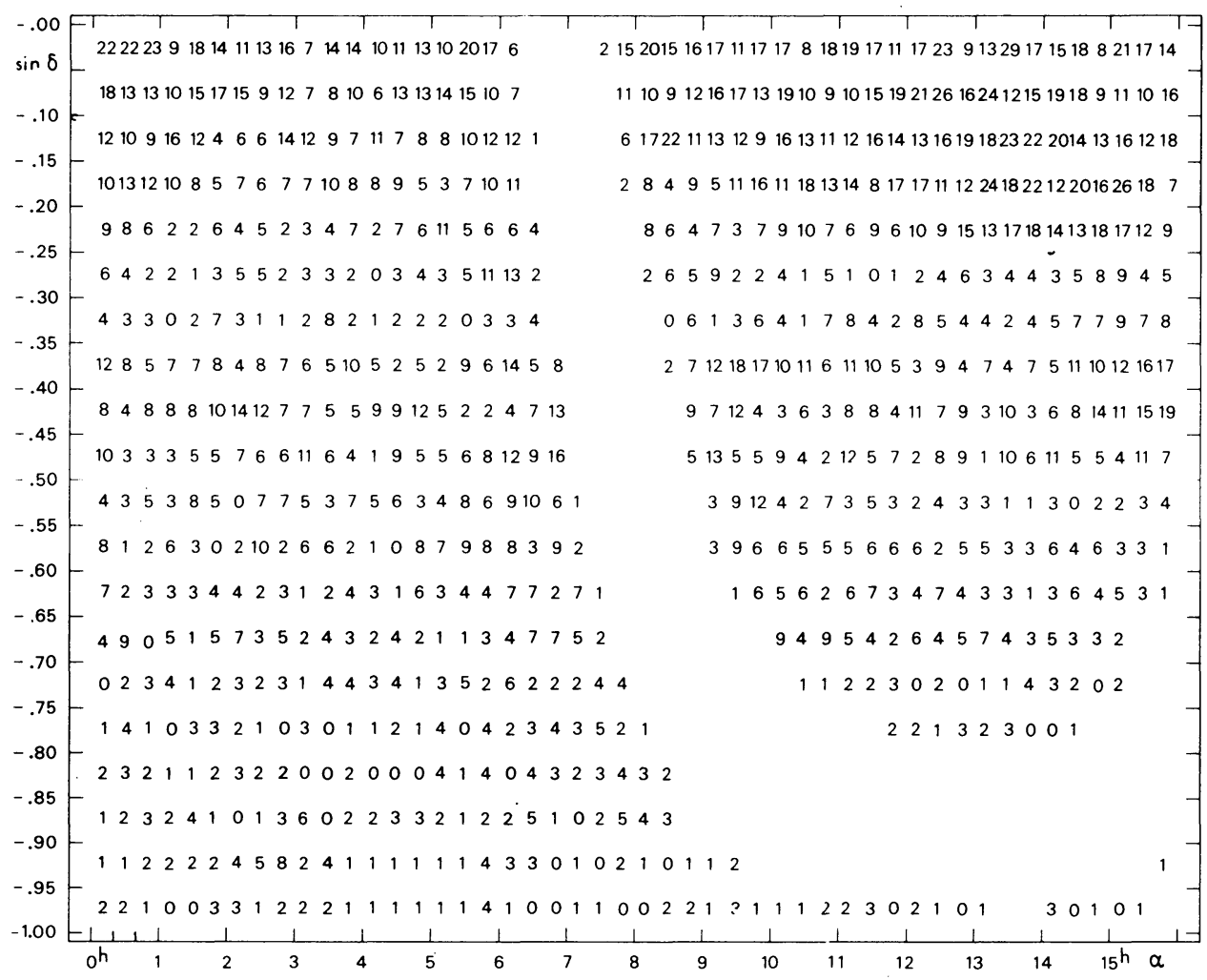

Fig. 1. Number of stars, south of $\delta=0^{\circ}$, zone $|b|>10^{\circ}$, (without variables or close doubles) for which 8 or more entries in the GFH or for which 5 or more in the Hamburger Index is found. 
in Figure 1. As is to be expected, most of these stars are situated in a zone within $15^{\circ}$ south of the equator.

For the greatest portion of the sky, the distribution of good-history stars is too poor to make use of them in connection with galaxies. It should be remembered that the fundamental stars do not occur in the volumes searched.

We cannot remedy history so we have to proceed as fast as possible with covering the sky by photographic means.

\section{DISCUSSION}

Dieckvoss: I am in favour of omitting observations made before 1900 , the errors of old meridian catalogues being as they are.

Eichhorn: I think that the question of the value of old catalogues has been oversimplified in the past. The question of whether an old catalogue can be salvaged by determining the systematic errors of the positions in it depends on a number of parameters, among others the homogeneity of the errors and the number of stars in the catalogue in question. It is the accuracy with which the systematic errors can be determined which is most important for utilizing an old catalogue. Brosche's work has been an important step in this direction. The statistical analysis is complicated, and we are now in a position to use a better criterion for using or not using a catalogue than whether its epoch is before or after 1900 .

Van Herk: In 1845 a paper was published in which observations up to 1843 had been used to demonstrate the non-rectilinear motion of Sirius. The period of the 'wobble' was predicted to be very close to 50 years (we have it now 49.99) and the amplitude was estimated about 5 to $10 \%$ in error of the present value. It was Bessel's paper, and he could not have done it if the observations had been as bad as all that. 